\title{
ALGEBRAIC AUTOMORPHISM GROUPS OF PRO-AFFINE ALGEBRAIC GROUPS
}

\author{
DONG HOON LEE
}

\begin{abstract}
ABSTRACr. We study the maximum connected algebraic subgroup of automorphisms of certain pro-affine algebraic groups.
\end{abstract}

Introduction. For a pro-affine algebraic group $G$ over an algebraically closed field $F$ of characteristic 0 , let $W(G)$ denote the group of all automorphisms of $G$. Although the group $W(G)$ may not be given a pro-affine algebraic group structure, Hochschild in [4] introduced the notion of algebraic subgroups of $W(G)$ formulated in terms of the Hopf algebra of polynomial functions and showed, among other things, that if $G$ is an affine algebraic group, then there is a connected algebraic subgroup of $W(G)$, which is the maximum in the sense that it contains every connected algebraic subgroup of $W(G)$. This result, however, does not extend to the pro-affine case as an example in [4] shows. We will call a pro-affine algebraic group $G$ an (MC)-group if $W(G)$ contains the maximum connected algebraic subgroup.

In this paper, we consider the question of when a pro-affine algebraic group $G$ is an (MC)-group. Our result (Theorem 1 in \$4) states that if the unipotent radical of $G$ is an (MC)-group, then so is $G$. This sharpens some of the results in [4]. Our study depends on the technique and results of [4] and also of [7]. We also show that an affine algebraic reductive group $G$ over a field of characteristic 0 is conservative if and only if $\operatorname{Int}(G)$ is of finite index in $W(G)$ (Theorem 2 in $\S 5$ ). This generalizes Theorem 2.1 in [7].

1. General properties and notations. We begin by recalling some definitions and results from [4] and [5]. Let $F$ be an algebraically closed field $F$ of characteristic 0 , and let $G$ be a pro-affine algebraic group over $F$ with Hopf algebra $Q(G)$ of polynomial functions on $G$ in the sense of [5]. We say that a subgroup $P$ of $W(G)$ is algebraic if $P$ can be given a pro-affine algebraic group structure over $F$ so that the map $G \times P \rightarrow G$ sending each $(x, \alpha)$ of $G \times P$ onto $\alpha(x)$ is a morphism of pro-affine algebraic varieties. In this case, the Hopf algebra $\mathcal{Q}(P)$ of polynomial functions on $P$ is generated (as an $F$-algebra) by the $F$-valued functions of the form $\rho / f$, with $\rho \in$ $\operatorname{Hom}(\mathbb{Q}(G), F)$ and $f \in \mathbb{Q}(G)$, where $\rho / f: P \rightarrow F$ is given by

Received by the editors April 10, 1979.

AMS (MOS) subject classifications (1970). Primary $20 \mathrm{G15}$.

Key words and phrases. Pro-affine, unipotent radical, conservative, Hopf algebra, reductive.

(C) 1980 American Mathematical Society 0002-9939/80/0000-0054/\$02.50 


$$
(\rho / f)(\alpha)=\rho(f \circ \alpha) \text { for } \alpha \in P .
$$

If $G$ is an affine (rather than pro-affine) group, then every algebraic subgroup of $W(G)$ is an affine algebraic group. We also know from [4, Theorem 2.1], that a subgroup $P$ of $W(G)$ is contained in an algebraic subgroup of $W(G)$ if and only if $\mathcal{Q}(G)$ is locally finite as a $P$-module, and if this condition is satisfied, the intersection $[P]$ of the family of all algebraic subgroups that contain $P$ is the smallest algebraic subgroup of $W(G)$ in which $P$ is algebraically dense. We will call $[P]$ the algebraic closure of $P$ in $W(G)$.

In addition to the notations already introduced above, the following are standard throughout: Let $G$ be a pro-affine algebraic group. Then $G_{0}$ will denote the connected component of 1 in $G$, and, for $x \in G, I_{x}$ will denote the inner automorphism of $G$ defined by $I_{x}(y)=x y x^{-1}$ for $y \in G$. For any subset $A$ of $G$, let $\operatorname{Int}_{G}(A)=\left\{I_{x}: x \in A\right\}$ and we will simply write $\operatorname{Int}(G)$ for $\operatorname{Int}_{G}(G)$.

2. A lemma. Let $G$ be a pro-affine algebraic group over an algebraically closed field $F$ of characteristic 0 , and let $G_{u}$ be the unipotent radical of $G$. For a subgroup $H$ of $G$, define $W(G)^{H}$ to be the subgroup of $W(G)$ consisting of $\alpha \in W(G)$ such that $\alpha(x)=x$ for all $x \in H$. Let $\zeta: W(G) \rightarrow W\left(G_{u}\right)$ denote the canonical map sending each $\alpha \in W(G)$ to the restriction $\alpha \mid G_{u} \in W\left(G_{u}\right)$.

LEMMA 1. If $Q$ is an algebraic subgroup of $W\left(G_{u}\right)$ and if $K$ is a maximal reductive subgroup of $G$, then $\zeta^{-1}(Q) \cap W(G)^{K}$ is an algebraic subgroup of $W(G)$.

Proof. Put $P=\zeta^{-1}(Q) \cap W(G)^{K}$. Then $Q(G)$ is locally finite as a $P$ module. To see this we argue as in [3, p. 104]. Thus the semi-direct decomposition $G=G_{u} \cdot K$ induces the tensor product decomposition

$$
\mathbb{Q}(G)=\mathscr{Q}(G)^{K} \otimes \mathbb{Q}(G)^{G_{u}}
$$

where $Q(G)^{H}$ for any subgroup $H$ of $G$ denotes the set of all $H$-fixed elements in $\mathcal{Q}(G)$. Clearly $P$ acts trivially on $\mathcal{Q}(G)^{G_{u}}$ and $P$ leaves $Q(G)^{K}$ invariant.

If we identify $\mathbb{Q}(G)^{K}$ with $\mathscr{Q}\left(G_{u}\right)$ via the restriction map $\mathscr{Q}(G)^{K} \rightarrow \mathscr{Q}\left(G_{u}\right)$ which is clearly an isomorphism, then the action of $P$ on $\mathscr{Q}(G)^{K}=\mathscr{Q}\left(G_{u}\right)$ is the transpose of the natural action of $\zeta(P)$ on $G_{u}$. Since $\zeta(P) \subset Q$, and since the action of $Q$ on $Q\left(G_{u}\right)$ is locally finite, it follows that the action of $P$ on $\mathcal{Q}\left(G_{u}\right)$ is locally finite, and this readily implies that $Q(G)$ is locally finite as a $P$-module.

Since $\zeta(P) \subset Q$ and since $Q$ is an algebraic subgroup, $\zeta$ maps the algebraic closure $[P]$ of $P$ into $Q$. Thus in order to show $[P]=P$, it is sufficient to show $[P] \subset W(G)^{K}$.

We first note that every element of $[P]$ leaves $K$ invariant. In fact, let $k \in K$ and let $f \in \mathbb{Q}(G)$ vanish on $K$. Then the polynomial function $k / f$ : $[P] \rightarrow F$ maps $P$ to $\{0\}$. Since $P$ is algebraically dense in $[P], k / f$ maps $[P]$ to $\{0\}$. Thus if $\alpha \in[P]$, then $f(\alpha(k))=(k / f)(\alpha)=0$ and since this holds for an 
arbitrary $f \in \mathbb{Q}(G)$ which vanishes on $K$, it follows that $\alpha(k) \in K$, proving that $\alpha(K) \subset K$.

The assignment of $\alpha \in[P]$ to $\alpha \mid K$ defines a map $\eta:[P] \rightarrow W(K)$. The image $\eta([P])$ is an algebraic subgroup of $W(K)$, and $\eta$ induces a morphism $[P] \rightarrow \eta([P])$ of pro-affine algebraic groups (cf. [4, Proposition 2.2]). It follows that $\operatorname{Ker} \eta$ is an algebraic subgroup of $W(G)$. But $\operatorname{Ker} \eta$ is easily seen to be identical with $P$, so that $P$ is an algebraic subgroup of $W(G)$.

3. Pro-affine reductive groups. We need the following lemma in $\S 4$.

LEMMA 2. Let $G$ be a reductive pro-affine algebraic group over an algebraically closed field $F$ of characteristic 0 . If $P$ is any algebraic subgroup of $W(G)$, then $P_{0}$ is contained in $\operatorname{Int}(G)$.

Proof. Assume first that $G$ is affine. We know from [4, Proposition 2.4] that $\operatorname{Int}(G) P$ is an algebraic subgroup of $W(G)$. Replacing $P$ by $\operatorname{Int}(G) P$ if necessary, we may assume that $\operatorname{Int}(G)$ is contained in $P$.

We claim that the Lie algebra $\mathcal{L}(P)$ of $P$ may be identified with a subspace of the $F$-linear space $Z^{1}(G, \mathcal{L}(G))$ consisting of all rational 1-cocycles of $G$ with values in the Lie algebra $\mathcal{L}(G)$ of $G$, on which $G$ acts by the adjoint representation. Let $\sigma \in \mathcal{L}(P)$. Thus $\sigma$ is an $F$-linear map $\sigma: \mathbb{Q}(P) \rightarrow F$ satisfying the usual differentiation condition.

For each $x \in G$, define $\sigma_{x}: \mathbb{Q}(G) \rightarrow F$ by $\sigma_{x}(f)=\sigma\left(x / x^{-1} \cdot f\right), f \in \mathbb{Q}(G)$. Here $y \cdot f$ for any $y \in G$ denote the left translate of $f$ by $y$, which is defined by $(y \cdot f)(z)=f(z y), z \in G$. Then $\sigma_{x} \in \mathcal{L}(G)$, and we have the relation $\sigma_{x y}=\sigma_{x}+\operatorname{Ad}(x)\left(\sigma_{y}\right)$ for $x, y \in G$. (See [7, pp. 146-148] for the detailed computation of the above and of others that follow below.) Thus the assignment $x \mapsto \sigma_{x}$ defines a cocycle $\sigma^{\prime} \in Z^{1}(G, \mathcal{L}(G))$. Since the functions $x / f$, together with their antipodes, generate $\mathbb{Q}(P)$ as an $F$-algebra, it follows that the $F$-linear map $\sigma \mapsto \sigma^{\prime}$ is an injection from $\mathcal{L}(P)$ into $Z^{1}(G, \mathcal{L}(G)$ ) under which we identify $\mathcal{L}(P)$ with an $F$-subspace of $Z^{1}(G, \mathcal{L}(G))$.

Consider now the morphism $\nu: G \rightarrow P$ of affine groups which is given by $\nu(x)=I_{x}, x \in G$. The image of the differential $\mathcal{L}(\nu): \mathcal{L}(G) \rightarrow \mathcal{L}(P)$ of $\nu$ is exactly the $F$-subspace $B^{1}(G, \mathcal{L}(G))$ of $Z^{1}(G, \mathcal{L}(G))$ consisting of all 1coboundaries of $G$. As $G$ is reductive, the cohomology group $H^{1}(G, \mathcal{L}(G)$ ) is trivial, and hence $\operatorname{Im}(\mathcal{L}(\nu))=B^{1}(G, \mathcal{L}(g))=Z^{1}(G, \mathcal{L}(G))$ contains $\mathcal{L}(P)$. Since $F$ is algebraically closed, $\nu(G)$ is open in $P$, and consequently we have $P_{0} \subset \nu(G)=\operatorname{Int}(G)$. This proves our assertion when $G$ is affine.

Next we assume that $G$ is an arbitrary reductive pro-affine algebraic group. Thus the Hopf algebra $Q(G)$ is a union of finitely generated sub-Hopf algebras $B$ of $Q(G)$, and each of such $B$ is in turn contained in a finitely generated $P$-stable sub-Hopf algebra of $\mathscr{Q}(G)$ [4, Proposition 2.3]. For each finitely generated $P$-stable sub-Hopf algebra $B$ of $Q(G)$, let $G_{B}$ denote the affine algebraic group whose elements are the restrictions of those of $G=$ $\operatorname{Hom}_{F \text {-alg }}(\mathbb{Q}(G), F)$ to $B$. Then clearly $G_{B}$ is reductive, and the canonical morphism $\pi_{B}: G \rightarrow G_{B}$ is surjective. For $\alpha \in P$, define $\alpha_{B} \in W\left(G_{B}\right)$ by the 
relation

$$
\alpha_{B}\left(\pi_{B}(x)\right)=\pi_{B}(\alpha(x)) \text { for all } x \in G .
$$

The assignment $\alpha \mapsto \alpha_{B}$ defines a group homomorphism $\eta_{B}: P \rightarrow W\left(G_{B}\right)$, and $\eta_{B}(P)$ is an algebraic subgroup of $W\left(G_{B}\right)$. As we have already seen in the affine case, the identity component $\eta_{B}\left(P_{0}\right)$ is contained in $\operatorname{Int}\left(G_{B}\right)$. Now let $\alpha \in P_{0}$. Thus there exists $z \in G_{B}$ such that $\alpha_{B}\left(x^{\prime}\right)=z x^{\prime} z^{-1}$ for all $x^{\prime} \in G_{B}$. Consider the nonempty set $T(B)=\left\{z \in G_{B}: \alpha_{B}=I_{z}\right\}$. If $z_{1}$ and $z_{2}$ are elements of $T(B)$, then $z_{1} x^{\prime} z_{1}^{-1}=z_{2} x^{\prime} z_{2}^{-1}$ for all $x \in G_{B}$, which readily implies that $z_{1}^{-1} z_{2}$ is central in $G_{B}$. This shows that $T(B)$ is identical with a coset of the center of $G_{B}$, and as such is closed in $G_{B}$. Suppose that $B_{1}$ and $B_{2}$ are finitely generated $P$-stable sub-Hopf algebras of $Q(G)$ with $B_{1} \geqslant B_{2}$. The canonical morphism $G_{B_{1}} \rightarrow G_{B_{2}}$ is a closed map and maps $T\left(B_{1}\right)$ into $T\left(B_{2}\right)$. Hence the standard projective limit theorem (see, e.g., [5, p. 1131]) may be applied to the $T(B)$ 's together with the closed maps $T\left(B_{1}\right) \rightarrow T\left(B_{2}\right)$ to conclude that the projective limit $Q$ of the $T(B)$ 's is nonempty. Now, let $y \in Q$. Then for all $x \in G$,

$$
\pi_{B}(\alpha(x))=\alpha_{B}\left(\pi_{B}(x)\right)=\pi_{B}(y) \pi_{B}(x) \pi_{B}(y)^{-1}=\pi_{B}\left(y x y^{-1}\right)
$$

for all $B$, and hence $\alpha(x)=y x y^{-1}$ for all $x \in G$, proving that $\alpha \in \operatorname{Int}(G)$.

4. Main theorem. For a pro-affine algebraic group $G$, the maximum connected algebraic subgroup of $W(G)$ (if it exists) is denoted by $W_{1}(G)$.

THEOREM 1. Let $G$ be a pro-affine algebraic group over an algebraically closed field $F$ of characteristic 0 . If the unipotent radical $G_{u}$ is an (MC)-group, then so is $G$.

Proof. Choose a maximal reductive subgroup $K$ of $G$, and let $A(K)$ denote the subgroup $\zeta^{-1}\left(W_{1}\left(G_{u}\right)\right) \cap W(G)^{K}$, using the notation of $\S 2$. By Lemma 1 , $A(K)$ is an algebraic subgroup of $W(G)$. Thus our assertion will follow as soon as we show that every connected algebraic subgroup $P$ of $W(G)$ is contained in $\operatorname{Int}_{G}\left(G_{0}\right) A(K)$.

Replacing $P$ by the algebraic subgroup $\operatorname{Int}_{G}\left(G_{0}\right) P$ if necessary, we may assume that $\operatorname{Int}_{G}\left(G_{0}\right) \subset P$. Using the conjugacy theorem of maximal reductive subgroups [3, Theorem 14.2], we obtain $P=\operatorname{Int}_{G}\left(G_{0}\right) \cdot A$, where $A$ is the subgroup of $P$ consisting of all $\alpha \in P$ leaving $K$ invariant.

Let $\eta: W(G) \rightarrow W(K)$ be the map obtained by composing the canonical map $W(G) \rightarrow W\left(G / G_{u}\right)$ with an isomorphism $W\left(G / G_{u}\right) \cong W(K)$ resulting from an isomorphism $G / G_{u} \cong K$. Then $\eta(P)$ is a connected algebraic subgroup of $W(K)$. Since $W_{1}(K)=\operatorname{Int}_{K}\left(K_{0}\right)$ by Lemma 2, $\eta(P)$ (and hence $\eta(A))$ is contained in $\operatorname{Int}_{K}\left(K_{0}\right)$. On the other hand, $\eta(\alpha)=\alpha \mid K$ for $\alpha \in A$, and $\eta$ maps the subgroup $\operatorname{Int}_{G}\left(K_{0}\right)$ of $A$ onto $\operatorname{Int}_{K}\left(K_{0}\right)$. We can therefore write $A=\operatorname{Int}_{G}\left(K_{0}\right) \operatorname{Ker}(\eta \mid A)$, and consequently $P=\operatorname{Int}_{G}\left(G_{0}\right) \operatorname{Ker}(\eta \mid A)$. Clearly $\operatorname{Ker}(\eta \mid A) \subset A(K)$, and we have $P \leqslant \operatorname{Int}_{G}\left(G_{0}\right) A(K)$, proving our assertion. 
REMARK. It is clear that under the hypothesis of Theorem $1, W_{1}(G)=$ $\operatorname{Int}_{G}\left(G_{0}\right) A(K)_{0}$.

Corollary 1. Let $G$ be as in the theorem. If $G_{u}$ is finite dimensional, then $G$ is an (MC)-group. In particular, every affine algebraic group is an (MC)-group.

Proof. If $G_{u}$ is finite dimensional, then $W\left(G_{u}\right)$ is an affine algebraic group, and is, in fact, isomorphic with the algebraic group $W(L)$ of all automorphisms of the Lie algebra $L$ of $G_{u}$. Thus $G_{u}$ is an (MC)-group, and $G$ is (MC) by the theorem.

RemarK. If $G$ is as in Corollary 1 , we can describe $W_{1}(G)$ fairly easily. Choose a maximal reductive algebraic subgroup $K$ of $G$. Applying Lemma 1 to $Q=W\left(G_{u}\right)$, we see that $W(G)^{K}$ is an algebraic subgroup of $W(G)$. It is also clear from the proof of Theorem 1 that every connected algebraic subgroup of $W(G)$ is contained in the algebraic subgroup $\operatorname{Int}_{G}\left(G_{0}\right) W(G)^{K}$. It follows that $W_{1}(G)$ is equal to $\operatorname{Int}_{G}\left(G_{0}\right)\left(W(G)^{K}\right)_{0}$, the identity component of Int $_{G}\left(G_{0}\right) W(G)^{K}$. The above description of $W_{1}(G)$ is given in [4] (see the proof of Theorem 4.2) when $G$ is a connected affine algebraic group.

COROLlaRY 2. Let $G$ be a connected pro-affine algebraic group over $F$. Then any normal algebraic subgroup $N$, which is either pro-finite or reductive and commutative, is central in $G$.

Proof. In both cases, $W_{1}(N)=\{1\}$ by Lemma 2. Define $\rho: G \rightarrow W(N)$ by $\rho(x)(n)=x n x^{-1}, n \in N$ and $x \in G$. Then $\rho(G)$ is a connected algebraic subgroup of $W(N)$, so that $\rho(G)=\{1\}$, which implies that $N$ is central in $G$.

5. Conservative reductive groups. We recall from [6] that an affine algebraic group $G$ over a field $F$ of characteristic 0 is said to be conservative if $\mathscr{Q}(G)$ is locally finite as a $W(G)$-module, and that if $G$ is conservative then the automorphism group $W(G)$ itself becomes an affine algebraic group in a natural way.

The following theorem was proved in [7] when $F$ is algebraically closed.

TheOReM 2. Let $G$ be a reductive affine algebraic group over a field $F$ of characteristic 0 . Then $G$ is conservative if and only if $\operatorname{Int}(G)$ is of finite index in $W(G)$.

Proof. Assume $F$ is not algebraically closed, and let $L$ be an algebraically closed field containing $F$ as a subfield. Consider the affine algebraic group $G^{L}$ over $L$ obtained from $G$ by extending the field $F$ to $L$. Then $\mathcal{L}\left(G^{L}\right)=$ $\mathcal{L}(G) \otimes_{F} L$.

Let Ad: $G \rightarrow G l_{F}(\mathcal{L}(G))$ (resp. Ad': $G^{L} \rightarrow G l_{L}\left(\mathcal{L}\left(G^{L}\right)\right)$ ) denote the adjoint representation of $G$ (resp. of $\left.G^{L}\right)$. Then $G l_{F}(\mathcal{L}(G))^{L}=G l_{L}\left(\mathcal{L}\left(G^{L}\right)\right)$, and by a result of Chevalley [2, p. 109, Proposition 4], $\overline{\operatorname{Ad}(G)^{L}}=\operatorname{Ad}^{\prime}\left(G^{L}\right)$, where $\overline{\operatorname{Ad}(G)}$ denotes the closure of $\operatorname{Ad}(G)$ in the algebraic group $G l_{F}(\mathscr{L}(G))$. This shows that $\overline{\operatorname{Ad}(G)}$ is the set of all $F$-rational points of $\operatorname{Ad}^{\prime}\left(G^{L}\right)$.

On the other hand, $\operatorname{Ad}^{\prime}\left(G^{L}\right)$ is the orbit of the affine group $G^{L}$ at 
$1 \in G l_{L}\left(\mathcal{L}\left(G^{L}\right)\right)$, where $G^{L}$ is viewed as a transformation group acting on the affine variety $G l_{L}\left(\mathcal{E}\left(G^{L}\right)\right)$ via the adjoint representation $\operatorname{Ad}^{\prime}$. That is, $\operatorname{Ad}^{\prime}\left(G^{L}\right)$ is identified with a homogeneous space of $G^{L}$. By [1, Corollary 6.4], the set $\overline{\operatorname{Ad}(G)}$ of $F$-rational points of $\operatorname{Ad}^{\prime}\left(G^{L}\right)$ is a union of finitely many orbits of $G$. It follows that $\overline{\operatorname{Ad}(G)} / \operatorname{Ad}(G)$, and hence $\overline{\operatorname{Int}(G)} / \operatorname{Int}(G)$, is finite.

Suppose now that $G$ is conservative. Then the map $\nu: G \rightarrow W(G)$, given by $\nu(x)=I_{x}, x \in G$, a morphism of affine algebraic groups, and, as we have seen in the proof of Theorem 2.1 in [7], the differential $\mathcal{L}(\nu): \mathcal{L}(G) \rightarrow$ $\mathcal{L}(W(G))$ is surjective. Since $F$ is of characteristic 0 , the closure of $\nu(G)=$ $\operatorname{Int}(G)$ is open in $W(G)$, and hence $\overline{\operatorname{Int}(G)}$ is of finite index in $W(G)$. Since $\operatorname{Int}(G)$ is of finite index in $\overline{\operatorname{Int}(G)}$, it follows that $\operatorname{Int}(G)$ is of finite index in $W(G)$.

The other implication in the theorem is clear from the fact that $\mathcal{Q}(G)$ is a locally finite $\operatorname{Int}(G)$-module and that $\operatorname{Int}(G)$ is a normal subgroup of $W(G)$.

\section{REFERENCES}

1. A. Borel and J.-P. Serre, Théorèmes de finitude en cohomologie galoisienne, Comment. Math. Helv. 39 (1964), 111-163.

2. C. Chevalley, Théorie des groupes de Lie, vol. 2, Groupes algébriques, Hermann, Paris, 1951.

3. G. Hochschild, Introduction to affine algebraic groups, Holden-Day, San Francisco, Calif., 1971.

4. __ Algebraic automorphism groups. Illinois J. Math. 19 (1975), 131-144.

5. G. Hochschild and G. D. Mostow, Pro-affine algebraic groups, Amer. J. Math. 91 (1969), 1127-1140.

6. __ Automorphisms of affine algebraic groups, J. Algebra 22 (1972), 535-543.

7. D. H. Lee, On the group of automorphisms of affine algebraic groups, Trans. Amer. Math. Soc. 237 (1978), 145-152.

Department of Mathematics, Case Western Reserve University, Cleveland, Oho 44106 